\title{
Introduzione alla modellizzazione matematica nella scuola secondaria di secondo grado
}

\section{Introduction to mathematical modelling in upper secondary school}

\author{
Antonella Moser \\ Liceo scientifico "A. Labriola" - Roma Lido, Italia \\ 凶moser_a@virgilio.it
}

Sunto / Nell'ambito della didattica della matematica, i modelli matematici sembrano avere scarsa attenzione, nonostante il loro crescente sviluppo e utilizzo nel mondo reale. Nella prassi didattica spesso si presenta un modello già costruito, chiedendo agli studenti di quantificare grandezze che vi compaiono per rispettare determinate condizioni. Rimane il dubbio che quest'unico approccio aiuti effettivamente a interiorizzare il concetto di modello e a capirne l'importanza e l'efficienza come strumento matematico.

Quest'esperienza didattica stimola gli studenti a costruire un modello matematico descrittivo di una situazione basata su un gioco competitivo per introdurre le peculiarità della modellizzazione matematica. Seppur circoscritta a un certo tipo di modello matematico, l'esperienza evidenzia i passi fondamentali per realizzare un qualsiasi modello. In particolare, si pone una certa attenzione a far sì che gli studenti siano il più possibile autonomi proprio nella costruzione del modello, in modo che possano sviluppare un approccio consapevole e critico alla modellizzazione matematica.

Parole chiave: modellizzazione matematica; modello descrittivo; variabili; gioco competitivo; scuola secondaria di secondo grado.
Abstract / Mathematical models are extensively used in many fields of the modern society, even though they seem to be poorly explored in mathematics teaching at secondary school. In teaching practice, often a predefined mathematical model is presented, and students are asked to calculate the values of the variables involved in order to meet specific conditions. However, it is still questionable whether this approach actually helps the students understand the concept of mathematical model and its importance and efficiency as a mathematical tool.

This teaching experience encourages students to construct a descriptive mathematical model of a situation based on a competitive game in order to introduce the peculiarities of mathematical modelling. Although limited to a certain type of mathematical model, the experience highlights the fundamental steps for constructing any model. In particular, some attention is paid to ensuring that students become as autonomous as possible precisely in the construction of the model, so that they can develop a conscious and critical approach to mathematical modelling.

Keywords: mathematical modelling; descriptive model; variables; competitive game; upper secondary school. 


\section{Introduzione}

È ormai evidente, al giorno d'oggi, l'importanza che i modelli matematici rivestono nell'analisi delle situazioni e dei fenomeni reali che ci circondano. A tal proposito, l'Accademia dei Lincei evidenzia che:

«Nella pratica reale della ricerca scientifica si lavora, di fatto, sempre con "modelli" [...]. Lo sviluppo di potenti tecniche numeriche e, recentemente, di metodi basati sull'intelligenza artificiale (come il machine learning), assieme all'utilizzo dei Big Data, ha rivitalizzato il problema della costruzione di modelli, ponendo nuove interessanti sfide».

(Accademia dei Lincei, 2019) ${ }^{1}$

In ambito educativo, anche le Indicazioni Nazionali italiane (Ministero dell'Istruzione, dell'Università e della Ricerca [MIUR], 2010) richiamano il tema dei modelli matematici tra gli obiettivi specifici di apprendimento già al termine del primo biennio del liceo scientifico: «Sarà approfondito in modo rigoroso il concetto di modello matematico, distinguendone la specificità concettuale e metodica rispetto all'approccio della fisica classica» (p. 340). Durante il secondo biennio lo studente «In relazione con le nuove conoscenze acquisite approfondirà il concetto di modello matematico» (MIUR, 2010, p. 341). $\mathrm{E}$, nel corso del quinto anno, «In relazione con le nuove conoscenze acquisite, anche nell'ambito delle relazioni della matematica con altre discipline, lo studente approfondirà il concetto di modello matematico e svilupperà la capacità di costruirne e analizzarne esempi» (MIUR, 2010, p. 341).

In alcuni libri di testo per la scuola secondaria di secondo grado² (Bergamini et al., 2017; Sasso \& Zanone, 2019), vengono presentati diversi problemi del tipo Realtà e Modelli che si riferiscono a modelli matematici. Questo tipo di problemi viene inserito nelle sezioni pratiche relative agli strumenti matematici utilizzati per modellizzare una situazione reale (esponenziali, equazioni differenziali, ricerca di massimi e minimi), ma è assente una sezione teorica che riguardi esclusivamente la modellizzazione matematica. Poiché le sezioni teoriche normalmente corrispondono ai temi affrontati nelle usuali programmazioni didattiche, può sembrare strano che tale sezione non sia presente. Nonostante il recente e notevole sviluppo dell'utilizzo dei modelli in molti campi della società moderna, sempre più orientata verso quelle che sono chiamate STEM disciplines (Science, Technology, Engineering and Math), e la breve parentesi di attenzione del MIUR con l'inserimento di tipologie di problemi basati su modelli matematici nell'esame di stato, perché sembra esserci ancora una certa reticenza a introdurre il tema della modellizzazione matematica nella programmazione di classe? Forse la maggior parte degli insegnanti non sono adeguatamente formati per trattare la disciplina dal punto di vista applicativo, in particolare mediante modelli matematici?

Esaminando i seminari dei più importanti convegni nazionali sulla didattica della matematica degli ultimi anni, ${ }^{3}$ o gli argomenti dei corsi per insegnanti indetti dall'AIRDM, ${ }^{4}$ sembra quasi di non scorgere traccia di questo contenuto disciplinare. È presente in convegni scientifici ${ }^{5}$ ove si illustrano esempi di applicazioni complesse in vari campi (non solo scientifici), ma senza alcun accenno al modo di insegnarli (e comunque si tratta di modelli sviluppati con strumenti matematici che si affrontano solo all'università, probabilmente incomprensibili per uno studente della scuola secondaria di secondo

1. https://sites.google.com/view/modellilincei2019/

2. La scuola secondaria di secondo grado in Italia dura cinque anni e corrisponde all'ultimo anno di scuola media e alla scuola media superiore o scuole professionali nel Canton Ticino.

3. Fra gli altri citiamo: XXXIII Convegno Nazionale Incontri con la matematica, novembre 2019 (BO); AIRDM: L'insegnamento della matematica tra procedure e concetti: la ricerca di un delicato equilibrio, Agosto 2019, Frascati (RM); Convegno Nazionale Educare alla razionalità: L'insegnamento della matematica e della logica nella scuola secondaria, Università di Torino, Maggio 2019 (TO); EDU-SIMAI: La matematica applicata incontra la scuola, Luglio 2018 (RM).

4. Associazione Italiana di Ricerca in Didattica della Matematica.

5. Per esempio: https://www.lincei.it/it/manifestazioni/genesi-dei-modelli-convegno 
grado). Se si esclude un tentativo all'interno di un convegno, dedicato proprio alla possibilità di trasferire la conoscenza dei modelli matematici dai laboratori alle aule didattiche (Natalini, 2013), solo durante l'anno scolastico 2019/2020, in piena pandemia, con le continue informazioni sulle previsioni dei modelli matematici per la diffusione del coronavirus, si è sviluppata una maggiore curiosità verso questo strumento e verso la possibilità di renderlo almeno parzialmente accessibile agli studenti mediante appositi laboratori.

Esula dallo scopo di quest'articolo il cercare di capire perché le Indicazioni Nazionali non sembrino essere osservate completamente e se proprio questo sia alla base delle difficoltà e perplessità non raramente dimostrate dagli studenti a riguardo dei modelli matematici anche alla fine del loro percorso scolastico nella scuola secondaria di secondo grado. Tali perplessità si riscontrano nell'incapacità di definire il modello matematico, nella manifestata assenza di conoscenza dei passi fondamentali per la relativa costruzione, di conoscenza delle differenti tipologie, di concezione dell'importanza e utilità dei modelli matematici. ${ }^{6}$

Eppure, alcuni modelli matematici sono estremamente semplici e a volte utilizzati senza riconoscerli. Come si potrebbe dunque introdurre la modellizzazione matematica nelle scuole secondarie di secondo grado? Una proposta metodologica efficace potrebbe essere quella di stimolare gli studenti a costruire un modello matematico semplice, ponendo attenzione ai prerequisiti che agevolano l'autonomia degli studenti durante tale costruzione; in quest'ottica, un esempio interessante è il modello descrittivo come strumento per rappresentare e descrivere quantitativamente situazioni reali o fenomeni naturali a loro vicini.

L'esperienza didattica qui proposta si focalizza proprio sulla costruzione di un modello matematico descrittivo di una situazione reale, basata su un gioco competitivo. La realizzazione del modello è preceduta da una fase introduttiva in cui vengono svolte alcune attività che favoriscono e verificano I'acquisizione dei prerequisiti. II messaggio che si vuole lasciare agli allievi con questa esperienza didattica è che ricorrere a un modello significa rispettarne una caratteristica che gli è peculiare (che lo rende fra l'altro estremamente versatile): la caratteristica di trovare una relazione fra i dati iniziali e finali del problema che sia applicabile per qualsiasi valore di detti dati; caratteristica che rappresenta una differenza sostanziale tra i passi che costituiscono un modello matematico rispetto a quelli relativi a un approccio risolutivo specifico per un determinato problema. Questa differenza è puntualizzata mediante un esempio pratico descritto nell'Allegato 1, che può eventualmente essere proposto e discusso con gli allievi a conclusione dell'attività di modellizzazione.

\section{Una versatile esperienza didattica: costruzione di un modello matematico descrittivo}

L'esperienza didattica descritta di seguito è stata realizzata in una classe quarta di una scuola secondaria di secondo grado (liceo scientifico tradizionale). Con opportune semplificazioni l'esperienza è adattabile anche ad altre classi a partire dalla classe seconda dello stesso ciclo di istruzione (Allegato 2).

Quanto realizzato è frutto di raffinamenti successivi di varie esperienze precedenti, condotte dall'autrice di questo articolo sempre nello stesso tipo di scuola. ${ }^{7}$ Si è cercato di porre una particolare attenzione nell'equilibrare ciò che si predispone per gli studenti e quello che essi stessi devono invece

6. L'autrice ha rilevato empiricamente tali perplessità, lavorando con studenti e studentesse alla fine del quinto anno in occasione di sostituzioni saltuarie di colleghi, con gruppi di studenti intenti a preparare i test di ingresso all'università e/o esami di analisi del primo anno universitario.

7. Le prime esplorazioni didattiche sono avvenute con gli alunni di tre scuole statali di Roma, due site in periferia (liceo scientifico "A. Labriola", liceo scientifico e linguistico "F. Enriques") e una in un quartiere residenziale (liceo classico e scientifico "Democrito"), negli anni scolastici dal 2014 al 2018 (una media di tre classi per anno scolastico). 
capire e realizzare da soli, cogliendo l'essenza stessa del modello matematico, e vivendo contemporaneamente dei momenti in cui hanno l'autentica sensazione di "afferrare un'idea". Un'idea propria, frutto di tentativi e analisi dei relativi risultati, che stimoli ulteriormente un'esplorazione autonoma. In una prima esplorazione del tema, si è cercato di sondare se la parte strettamente matematica fosse alla portata di studenti liceali. Da queste esperienze "brevi" di 3-4 ore-lezione, si è passati a effettuare esperienze più lunghe (7-8 ore-lezione) provando a far costruire agli studenti un modello matematico per la situazione proposta. Anche in queste ulteriori esperienze era stato necessario guidare in modo marcato il lavoro degli studenti. Si è deciso dunque di effettuare alcune premesse pratiche e teoriche all'esperienza vera e propria di costruzione di un modello. In particolare, si è pensato di sottoporre inizialmente gli studenti all'analisi delle caratteristiche rappresentative di un semplice dispositivo reale (la ruota idraulica) e alla sua possibile rappresentazione grafica semplificata. Inoltre, si è cercato di far capire ai discenti, attraverso un video esplicativo, la differenza fra parametri e variabili in una rappresentazione matematica di un fenomeno reale.

Nel corso dell'esperienza didattica, seppur circoscritta a un modello matematico descrittivo, si evidenziano comunque i passi fondamentali per realizzare qualsiasi modello. Si offre inoltre un modo per interiorizzare i concetti di variabile caratteristica di un fenomeno, di un processo, di un dispositivo, di una situazione reale, e variabile matematica. Grazie a questa prima semplice esperienza didattica, la proposta poi di modelli via via più complessi sarà probabilmente accolta con minore perplessità. Inoltre, al suo interno sono previsti dei passaggi che offrono spunti per potenziare il concetto di sequenza numerica e di formula ricorsiva.

Nello specifico, l'esperienza didattica consiste nella costruzione di un modello matematico molto semplice, descrittivo di una situazione reale che coinvolge facilmente gli studenti: un gioco competitivo fra squadre. Agli studenti si presenta il caso di una gara di corsa e abilità: la pumpkin race (Figura 1), una gara di zucche della tradizione americana. Tuttavia, potrebbe trattarsi anche di una generica gara a staffetta; il modello rappresentativo sarebbe lo stesso.

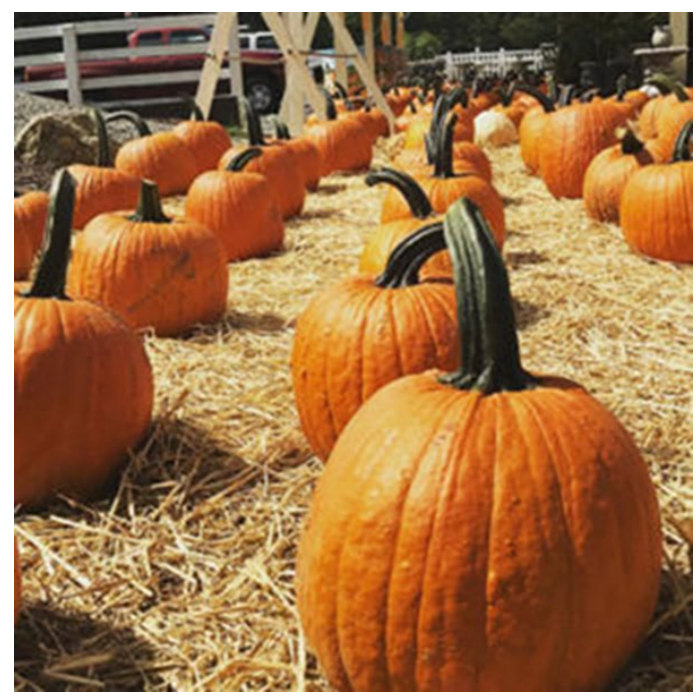

Figura 1. File ordinate di zucche predisposte per il gioco competitivo

Nella pumpkin race, si predispongono le zucche una dietro l'altra, in file parallele a una certa distanza da una linea di start, in modo che lo spazio fra una zucca e la successiva della stessa fila sia identico per tutte le zucche.

I partecipanti alla gara si dividono in squadre, ciascuna posta in prossimità di una fila di zucche e della 
linea di start. La gara consiste nel raccogliere più zucche possibili della propria fila, in un certo tempo fissato. Le zucche vanno raccolte in ordine sequenziale a partire dalla prima, inoltre, presane una, la si deve riportare alla linea di start e poi andare a prendere la successiva. Ogni giocatore della squadra deve prendere almeno tre zucche prima di farsi dare il cambio dal giocatore successivo.

Per ogni competizione, il tempo di durata della gara, la distanza fra le zucche, la distanza della prima zucca dallo start sono fissati a priori, ma potrebbero variare di anno in anno a causa delle condizioni atmosferiche e/o di esigenze organizzative.

La squadra che vince la gara può ottenere anche un premio speciale se sarà riuscita a stabilire il record di velocità media rispetto agli anni precedenti.

Alla fine di ogni competizione è quindi importante determinare la velocità media della sola squadra vincitrice (calcolando i metri effettivamente percorsi da tutti i componenti della squadra e rapportandoli al tempo avuto a disposizione, ovvero alla durata stabilita per la gara).

L'attività didattica proposta agli studenti consente, sulla base della gara appena descritta, di sviluppare per gradi un modello matematico, rappresentativo della gara ed efficiente nel determinare proprio i metri percorsi da una squadra e quindi la relativa velocità media sostenuta.

\subsection{Fasi e caratteristiche dell'esperienza didattica}

In questo paragrafo si riportano schematicamente le fasi dell'esperienza didattica, le finalità e i contenuti coinvolti, la modalità didattica adottata e le procedure valutative attivate.

\section{Fasi dell'esperienza}

1. Fase introduttiva:

- Introduzione al concetto di modello matematico.

- Introduzione ai concetti di caratteristiche di una situazione reale e di analisi di dette caratteristiche, mediante l'esame di esempi concreti in cui si evidenzia anche la differenza fra individuazione delle variabili caratteristiche e loro analisi.

- Analisi della differenza fra parametri e variabili mediante un esempio concreto.

- Presentazione della situazione reale che si vuole rappresentare in termini matematici.

2. Fase realizzativa:

- Individuazione e analisi delle caratteristiche della situazione reale.

- Individuazione di variabili matematiche da associare alle caratteristiche della situazione reale.

- Realizzazione di un modello grafico che schematizzi la situazione.

- Ricerca di modi intuitivi, empirici per determinare i valori delle variabili matematiche, iniziando da casi particolari (corrispondenti a valori predeterminati di alcuni parametri).

- Ricerca di una modalità matematica per determinare gli stessi valori, che sia più efficace di quella empirica, a partire dai primi risultati calcolati empiricamente.

- Ricerca di una relazione diretta fra le variabili matematiche.

- Generalizzazione della relazione individuata.

3. Fase conclusiva:

- Analisi del modello matematico generato.

- Rielaborazione verbale, strutturale dell'esperienza.

\section{Finalità}

Preparare gli studenti a sviluppare in maniera consapevole e critica l'approccio alla modellizzazione matematica.

Contenuti

- Relazioni, funzioni parametriche. 
- Parametri, variabili.

- Sequenze numeriche.

- Successioni ricorsive e formule ricorsive.

- Modelli matematici.

Competenze attivate

- Analizzare dati e situazioni reali.

- Rappresentare graficamente situazioni reali.

- Argomentare strategie e scelte.

- Costruire e utilizzare modelli descrittivi.

- Interpretare e valutare i risultati matematici ottenuti con il modello all'interno del contesto reale di partenza.

\section{Modalità operative}

Tempi. L'attività didattica completa (fase introduttiva, realizzativa e conclusiva) richiede dalle 8 alle 10 ore di lezione (in base alle competenze logico-matematiche del gruppo classe) più qualche ora pomeridiana extrascolastica di studio individuale e/o di gruppo.

Metodi. Sono stati alternati due metodi didattici. A volte si forniva un certo tempo a tutti gli studenti per arrivare a formulare una risposta a quanto richiesto, chiedendo di descrivere i risultati a cui erano giunti sul quaderno per poi controllarli singolarmente. Questa strategia ha fatto sì che gli studenti con competenze più avanzate non interrompessero il ragionamento dei compagni e che ognuno riflettesse con i tempi che gli sono propri. Altre volte si è lasciato che gli studenti esprimessero a turno le loro proposte così come le avevano maturate, per poi discuterle collettivamente.

Si è proposto prima un modello semplificato, avendo fissato a priori i valori di alcuni parametri per poi aumentare la difficoltà e giungere a un modello generale.

Gli studenti hanno spesso lavorato in piccoli gruppi, specie nella fase realizzativa del modello grafico semplificato e di quello matematico, e nelle fasi scritte di rielaborazione dell'esperienza. Per la ricerca della sequenza rappresentativa dei metri percorsi per prendere le varie zucche, gli studenti hanno invece lavorato in modalità individuale. Continue rielaborazioni verbali individuali venivano richieste, alla fine di ogni fase, per consolidare quanto appreso durante la fase stessa.

\section{Modalità di valutazione}

La valutazione dell'esperienza didattica è stata realizzata mediante prove di diverso genere, sia durante che alla fine dell'esperienza. Sono stati realizzati vari momenti valutativi, in particolare: per valutare la comprensione della parte introduttiva è stato utilizzato un test con domande a risposta aperta (Allegato 3), con lo scopo di capire in particolare se gli studenti fossero in grado di inquadrare storicamente la nascita dei modelli matematici, se sapessero darne una definizione, e se fossero in grado di distinguerne le varie tipologie. Per le sequenze numeriche, sono stati somministrati esercizi sul riconoscimento di regolarità di sequenze con eventuale associazione di una formula ricorsiva, scelti fra quelli riportati in un testo scolastico dedicato alla preparazione della prova di matematica dell'Esame di Stato di fine liceo scientifico (Bergamini et al., 2015). L'insegnante ha impostato inoltre, come richiesta di risoluzione del problema, di determinare la velocità media della squadra vincitrice, mediante la ricerca della relazione fra metri percorsi e numero di zucche prese.

Alla fine dell'esperienza sono state richieste agli studenti delle rielaborazioni schematiche della fase realizzativa, da svolgere in piccoli gruppi, con lo scopo di capire se gli studenti avessero compreso la necessità dei vari passaggi realizzati durante l'esperienza; se avessero appreso quali sono le fasi alla base della costruzione di un modello matematico e l'efficacia di ricorrere a un modello per descrivere una situazione reale. 


\subsection{Fase introduttiva}

2.2.1 Lezione 1: introduzione al concetto di modello matematico, cenni ad alcune definizioni storiche e alle diverse tipologie di modelli

In questa prima lezione, si è cercato di introdurre il concetto di modello matematico a partire da alcune definizioni storiche per poi concludere descrivendo brevemente le diverse tipologie di modelli. È stata utilizzata una lezione frontale, supportata dalla visualizzazione di alcune diapositive realizzate dall'insegnante (Allegato 4), in cui sono riportate per intero diverse citazioni e presentati in modo schematico alcuni concetti chiave e le tipologie di modelli.

Fra le tante definizioni di modello e di modello matematico, si è preferita quella di Valeriano Comincioli (2004):

«Un modello, dal latino modulus (diminutivo di modus, misura), è un oggetto, o un concetto, che è usato per rappresentare qualcosa d'altro. In particolare, un modello matematico è un modello che ha come componenti concetti matematici, come costanti, variabili, funzioni, equazioni. [...] La parola "modello" implica originariamente un cambiamento di scala nella sua rappresentazione. Attualmente, tale significato rimane nel senso che un modello ad esempio matematico, rappresenta un cambiamento sulla scala di astrazione: per ottenere il modello certi particolari vengono rimossi e vengono introdotte delle semplificazioni».

(Comincioli, 2004, p. 1)

Si è poi fatto notare agli studenti come la parola "modello" ci riporti alla sua definizione nelle arti, come di una rappresentazione in qualsiasi materiale di un oggetto reale (esistente o da realizzare). Questa idea si collega infatti a una prima definizione di Giorgio Israel (2003) che considera ingenua:

«Nel nostro caso dobbiamo costruire il modello con una "materia" affatto speciale: la matematica. Disponiamo in tal modo di una definizione che deriva dal significato testuale delle parole: il modello matematico è una rappresentazione, in linguaggio matematico, di un aspetto della realtà, sia che esista già sia che si tratti di realizzarlo. [...] Di fatto queste due parole (matematica applicata ma soprattutto "modelli matematici") cominciarono ad essere usate sistematicamente quando entrò in crisi non soltanto la concezione meccanicistica ${ }^{8}$ ma anche la concezione unitaria ${ }^{9}$ della scienza [...] Già in una conferenza del 1901 il matematico italiano Vito Volterra (1860-1940) descrisse in modo assai chiaro il nesso fra la crisi della scienza classica e l'introduzione dei "modelli"».

(Israel, 2003, pp. 6-7)

In seguito, si è chiarito come i modelli matematici costituiscano un potente strumento per rappresentare e descrivere quantitativamente un fenomeno naturale, o un'attività reale, per visualizzare le proprietà di una data teoria, e per rappresentare il progetto di un modello reale di un artefatto tecnologico (in questi casi si parla di modelli descrittivi). I modelli matematici sono inoltre utilizzati per le scienze sociali come modelli di controllo, cioè come modelli che possano definire i comportamenti più idonei in certe circostanze secondo criteri etici, di pubblica utilità (i modelli di controllo vengono utilizzati nelle applicazioni più svariate, per esempio nella teoria delle code in telecomunicazioni); si tratta dunque di modelli matematici che permettono di intervenire sulla realtà.

Nella breve descrizione delle tipologie di modelli si è ritenuto utile accennare anche al tema dei modelli deterministici. I pochi esempi riportati in molti libri di testo sono proprio di questo tipo (ad

8. Secondo questa concezione tutti i fenomeni dell'Universo sono riconducibili al moto dei corpi e poiché questi moti sono retti da formule matematiche ben determinate, queste formule possono costituire una base di rappresentazione anche per i fenomeni dell'Universo.

9. Le differenti parti della scienza debbono essere collegate e coerenti fra loro e formare una costruzione unitaria, all'interno della quale il posto preminente è riservato alla meccanica. 
esempio, un isotopo radioattivo che decade nel tempo riducendo la sua massa, oppure l'evoluzione di una popolazione di batteri nel tempo). La maggior parte dei modelli descrittivi o di controllo sono deterministici, ossia descrivono l'evoluzione di un fenomeno, di un'attività reale, di una situazione, in modo che noto il valore di certe variabili di stato (rappresentative del fenomeno a un certo momento) si riesca a determinare il loro valore futuro e passato in modo univoco. A questo proposito, Giorgio Israel (2003) specifica:

«[...] diremo che il processo evolutivo di un sistema reale è deterministico se esso è governato da una legge strettamente causale. Le cause che agiscono sul sistema considerato determinano in modo univoco la sua evoluzione, senza alcuna alternativa possibile [...], i matematici enunciano questo principio dicendo che le condizioni iniziali del sistema determinano in modo univoco la sua evoluzione nel tempo».

(Israel, 2003, pp. 72-73)

Questa breve presentazione si è poi conclusa con un accenno a un'altra tipologia di modello: i modelli stocastici. Le caratteristiche di un fenomeno, o di un'attività reale, che si vuole rappresentare potrebbero variare in modo casuale; in questo caso si utilizzano modelli stocastici (particolarmente usati, ad esempio, nel mondo della finanza).

Parlando di questa tipologia di modelli si sarebbe potuto specificare che esistono altri casi intermedi rappresentabili proprio attraverso un modello a metà fra quelli deterministici e quelli stocastici (modelli semi-deterministici), ma non si è voluta appesantire questa introduzione con ulteriori informazioni teoriche, che potranno tuttavia costituire un approfondimento futuro per gli studenti.

\subsubsection{Lezione 2: esempio di analisi di variabili e rappresentazione semplificata di un artefatto rea- le; studio del rendimento di una ruota idraulica}

La ruota idraulica è uno dei primi esempi di uno studio sistematico, prima empirico e poi matematico, dovuto all'esigenza di confrontare il rendimento delle ruote idrauliche verticali alimentate "da sotto" o "da sopra".

In generale, il rendimento di un motore è una variabile fisica espressa dal lavoro effettuato per unità di energia utilizzata. In termini matematici, esso è indicato dal rapporto fra il lavoro svolto sfruttando una certa quantità di energia e detta quantità di energia. L'idea di capire a parità di energia sfruttabile (ad esempio l'energia cinetica o potenziale di un corso d'acqua) quali siano le variabili fisiche che possono incidere per aumentare il lavoro effettuato (numeratore della frazione corrispondente al rapporto), e dunque il rendimento della macchina, può far intuire agli studenti cosa si intenda per analisi delle caratteristiche fisiche e far intravvedere la stretta connessione fra variabile fisica e variabile matematica. Nella sperimentazione sono state presentate due immagini di ruote, rispettivamente da sotto (Figura 2) e da sopra (Figura 3):

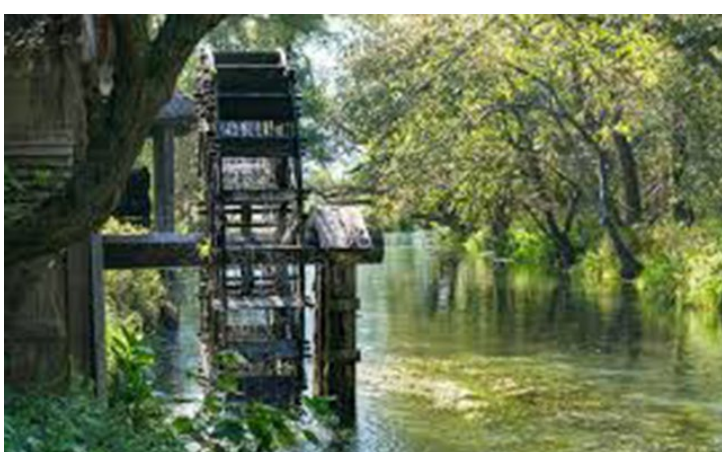

Figura 2. Ruota da sotto.

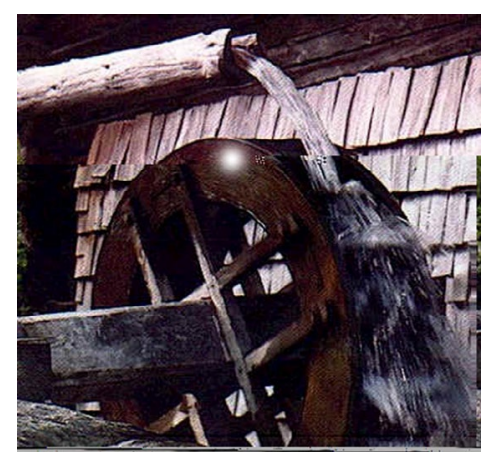

Figura 3. Ruota da sopra. 
Si è poi spiegato agli studenti che per studiare le caratteristiche fisiche che ne influenzano il rendimento è più adatto analizzare un modello grafico semplificato che rappresenti le ruote.

La docente ha dunque presentato due corrispondenti rappresentazioni, rispettivamente per il primo (Figura 4) e secondo tipo di ruota (Figura 5):

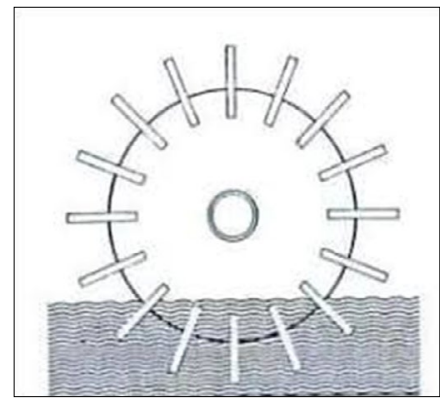

Figura 4. Schema ruota da sotto

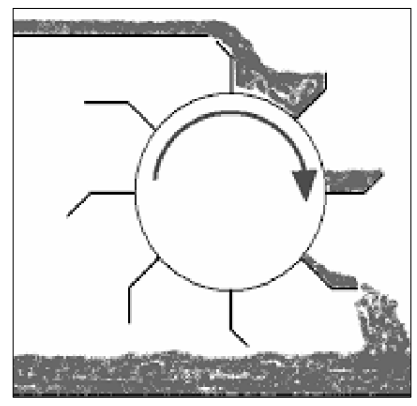

Figura 5. Schema ruota da sopra.

Si è poi chiesto agli studenti, in modalità di discussione collettiva, di individuare le possibili caratteristiche fisiche, reali che incidono sul rendimento della ruota. Tali variabili sono state chiamate variabili caratteristiche.

Nell'elencarle, gli studenti hanno nominato tutte le caratteristiche importanti, in modo ridondante, assieme ad altre irrilevanti e mischiando quelle tipiche della ruota da sotto con quelle della ruota da sopra (Figura 6).

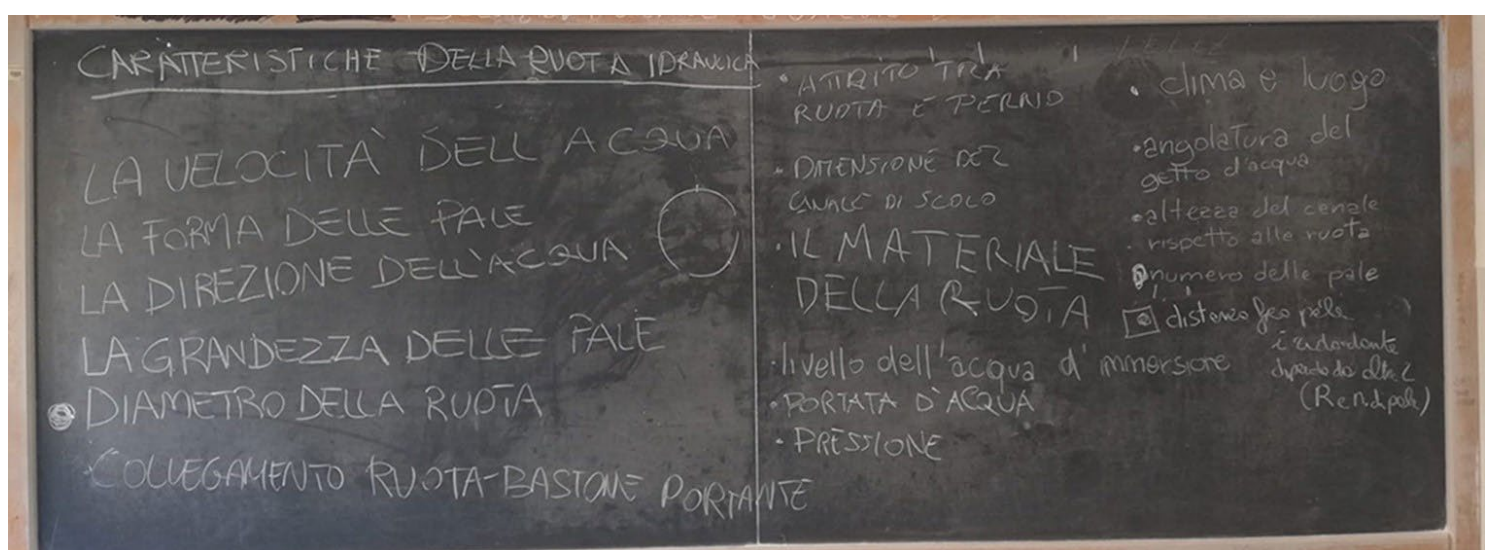

Figura 6. Variabili caratteristiche della ruota.

Si è dunque posta l'attenzione al fatto che aver individuato le variabili che influenzano il rendimento di questo motore primo non significa averne effettuato un'analisi.

L'insegnante ha quindi inizialmente invitato gli studenti a capire quali variabili dipendessero dalla situazione fisica in cui la ruota avrebbe operato (velocità del corso d'acqua e clima, ad esempio) e quali invece rappresentano delle scelte costruttive (raggio della ruota, numero di pale, distanza fra le pale). Fra queste ultime un'ulteriore riflessione ha portato a selezionare solo quelle che costituiscono un'effettiva scelta (dunque, escludendo per esempio l'attrito fra il perno centrale e l'asse della ruota). L'ultimo passo dell'analisi è stato capire fra le variabili selezionate quali fossero realmente indipendenti. L'insegnante ha proseguito con un esempio: fissato il raggio della ruota, il numero di pale e la distanza fra le pale non costituiscono due variabili indipendenti. II numero di pale, infatti, si ottiene 
dividendo la misura della circonferenza per la distanza fra le pale, viceversa la distanza fra le pale si ottiene dividendo la circonferenza per il numero di pale (formula inversa).

Si è introdotto così il concetto di dipendenza fra variabili.

Di seguito il risultato della selezione effettuata collettivamente e riordinata dall'insegnante:

- raggio della ruota;

- distanza fra le pale;

- lunghezza delle pale;

- dimensioni della concavità delle pale;

- (per la ruota da sotto) parte della ruota immersa nell'acqua;i10

- (per la ruota da sopra) altezza a cui posizionare il canale di scolo rispetto alla ruota.

Si è fatto riflettere gli studenti sull'importanza di trovare delle relazioni matematiche fra le variabili individuate e la potenza o il rendimento, in modo che si potesse determinare con buona approssimazione a priori (prima di costruire la ruota e verificarne il funzionamento) quali dovessero essere i valori ottimali corrispondenti al rendimento massimo. D'altro canto, questi valori ottimali nascono a volte da esigenze contrastanti. La docente ha chiesto agli studenti se il canale di scolo, ad esempio, dovesse essere posizionato abbastanza in alto rispetto alla ruota per sfruttare al meglio l'energia potenziale dell'acqua o se questa scelta non avesse dei risvolti negativi a parità di quantitativo d'acqua disponibile. A questo proposito, gli studenti sono rimasti inizialmente interdetti, per poi dire che troppo in alto ci sarebbe stata più dispersione di acqua specie in condizioni di vento. L'insegnante ha poi concluso spiegando che le ruote da sopra si utilizzano per salti naturali non superiori ai $6 \mathrm{~m}$. Se si costruisce il canale di scolo, si preferisce un'altezza molto ridotta (inferiore a un metro) che dipende dalla pressione con cui l'acqua arriva al salto.

\subsubsection{Lezione 3: analisi della differenza fra parametri e variabili; esempio pratico di utilizzo}

Un modello di solito è progettato per definire relazioni fra grandezze che possono variare durante una situazione, un esperimento, o lo studio di un caso. Per definire la relazione fra queste grandezze variabili se ne utilizzano anche alcune di altro tipo, i parametri, che rimangono costanti durante lo studio, ma che contribuiscono a determinare le variazioni delle prime.

Nel corso delle lezioni ordinarie, l'insegnante aveva notato come gli studenti avessero chiara la distinzione fra parametri e variabili nello studio dell'algebra (nelle equazioni parametriche, ad esempio, dimostravano di sapere che $x$ è la variabile e $k, a, b, c$ sono dei parametri) ma che facessero molta confusione fra questi due tipi di grandezze nei contesti applicativi. Venendo spesso denominate tutte le grandezze con lettere diverse da $x$, cui di volta in volta corrispondono dei valori, spesso gli studenti consideravano i termini "parametri" e "variabili" come sinonimi.

La docente ha quindi dedicato una lezione per chiarire la differenza fra questi due tipi di grandezze, tramite l'analisi di un video specifico, percependone in seguito l'efficacia. ${ }^{11}$

Il video riguarda la conservazione dell'energia meccanica ed è realizzato da Zanichelli12 (Figura 7).

10. Questa variabile è stata curiosamente individuata prontamente da uno dei più giovani studenti.

11. Durante la costruzione del modello, infatti, gli studenti sono riusciti a utilizzare i termini "parametri" e "variabile" in modo consapevole.

12. Il video è disponibile al seguente link: https://www.youtube.com/watch?v=bRqQRRXxx3| 


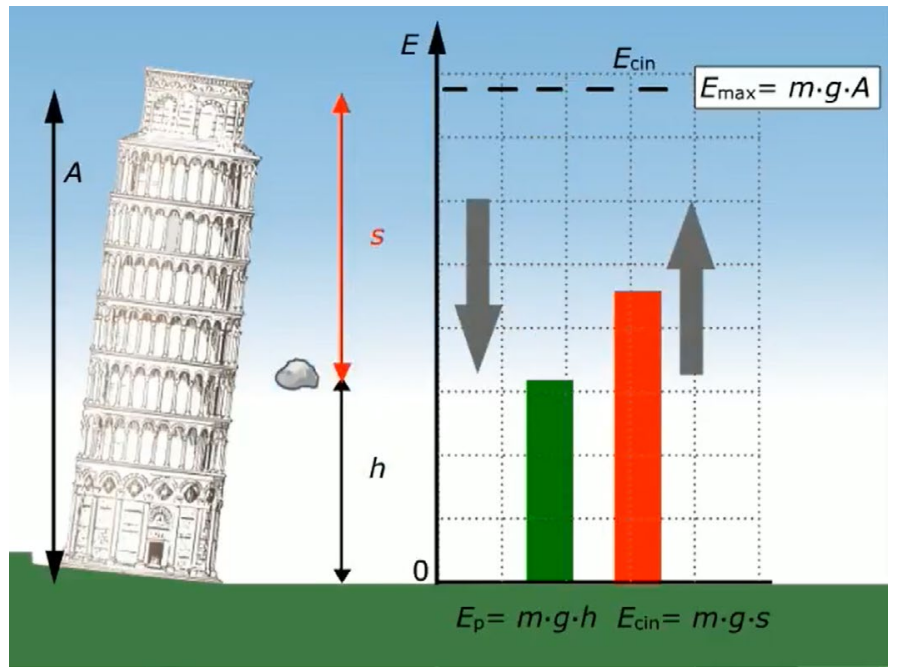

Figura 7. Immagine video Zanichelli La conservazione dell'energia meccanica.

In questo video si studia il caso di un sasso lanciato dall'estremità della Torre di Pisa e si analizza la variazione dell'energia cinetica e dell'energia potenziale man mano che il sasso percorre uno spazio $s$ verso il basso e di conseguenza varia la sua quota $h$ rispetto al suolo. L'energia potenziale massima si ha quando il sasso è in cima alla torre e quindi $E_{\max }=m \cdot g \cdot A$, dove si è posta pari ad $A$ l'altezza della torre e quindi di lancio.

In seguito alla visione del video, I'insegnante ha chiesto agli studenti di notare l'utilizzo di lettere minuscole per lo spazio e la quota, e di una lettera maiuscola per indicare l'altezza di lancio. Alla richiesta di dare una spiegazione di tale scelta, sono rimasti abbastanza interdetti. Molti non hanno saputo dire nulla. Alcuni hanno detto: «È indifferente, si tratta di una differenza casuale». Facendo notare loro che la scelta non è casuale ma è voluta per distinguere quelli che di fatto sono due tipi di grandezze diversi, uno studente dopo qualche minuto ha affermato che: «ll sasso poteva essere lanciato anche da un ponte alto meno della torre ma lo studio del caso rimaneva uguale». Gli è stato chiesto quindi che conclusione traesse da questa osservazione. Ha detto: «A sarebbe stata l'altezza del ponte ma di fatto non cambiava nulla della spiegazione successiva. Sarebbe stato solo un valore iniziale diverso da attribuire ad $A$ ». È stata sfruttata quest'ultima considerazione per rimarcare il fatto che $A$ poteva assumere valori diversi se corrispondeva all'altezza di una torre o di un ponte, ma che fissato il caso e quindi il suo valore iniziale questo poi nel corso di tutto lo studio non cambiava, pur restando un fattore che influenza il valore dell'energia meccanica totale. Infine, si è chiesto: «E quindi la differenza con se $h$ ?». Uno studente diverso ha concluso che: «s e $h$ durante la caduta variano, $A$ no».

Dopo aver verificato oralmente che questa differenza fosse chiara per tutti, l'insegnante ha fornito agli studenti una definizione più formale: un parametro è un ente matematico che può assumere vari valori a seconda dei casi, ma il suo valore viene stabilito e rimane costante per tutto lo studio di ogni singolo caso, sebbene il parametro non sia una costante (come il numero di Nepero e, come la costante di gravità $G$ che assumono lo stesso valore in qualunque contesto); le variabili invece assumono valori che variano durante lo studio del caso.

\subsubsection{Lezione 4: descrizione dell'attività che si vuole rappresentare in termini matematici}

Agli studenti è stata descritta la gara di zucche, come riportato nella parte introduttiva di questo paragrafo (par. 2), evidenziando il problema di calcolare la velocità media della squadra vincitrice per stabilire se risulti un valore migliorativo rispetto a quelli degli anni precedenti e si debba quindi assegnare il premio speciale. 
Agli studenti è stato lasciato il compito di dedurre che, per fare ciò, è sufficiente conteggiare il numero di zucche raccolte. Infatti, la velocità media sostenuta dalla squadra vincitrice si calcola mediante il seguente rapporto:

$v_{m}=\frac{\text { Spazio percorso }}{\text { Tempo impiegato }}$

Tempo impiegato $=T$ tempo gara fissato a priori per ogni gara

Come calcolare quanti metri ha percorso la squadra vincitrice? Considerando il numero di zucche prese, la distanza fra una zucca e la successiva della stessa fila, e la distanza della prima zucca dallo start. Ciò significa dover determinare quanto spazio si deve percorrere per arrivare a prendere l'ultima zucca raccolta dalla squadra vincitrice (se ha preso 10 zucche, occorre determinare quanto spazio bisogna percorrere per arrivare a raccogliere la decima zucca).

A questa conclusione si è giunti attraverso una discussione collettiva in cui il ruolo dell'insegnante è stato solo quello di orientare la riformulazione delle eventuali soluzioni errate degli studenti. Alcuni studenti, per esempio, hanno semplificato il calcolo moltiplicando il numero di zucche per la distanza fra le zucche e poi il risultato per 2 , dimenticando che la prima fosse a una distanza diversa dalla linea di partenza.

Vista l'erroneità delle soluzioni intuitive per calcolare lo spazio percorso, si è proposto di rappresentare la gara in termini grafici e matematici riscrivendo in chiaro l'obiettivo alla LIM:

Individuare quanti metri occorre percorrere per prendere una determinata zucca, da cui calcolare rapidamente quanti metri abbia percorso una squadra che abbia colto un certo numero di zucche (e quindi riuscire a determinare la velocità media sostenuta).

\subsection{Fase realizzativa del modello matematico}

\subsubsection{Lezione 5: individuazione e analisi delle caratteristiche dell'attività}

Dopo aver richiamato brevemente la fase introduttiva relativa al concetto di caratteristica di una situazione e di variabile matematica, gli studenti sono stati invitati a individuare le caratteristiche della situazione in esame tramite una riflessione prima individuale e poi una discussione collettiva.

In generale gli studenti hanno proposto:

- numero di zucche disposte su ogni fila;

- distanza della prima zucca dallo start;

- distanza fra una zucca e l'altra;

- posizione della zucca rispetto alle altre (prima, seconda, ...);

- numero di concorrenti per squadra;

- estensione del campo di gioco;

- tempo disponibile per la gara;

- metri da percorrere per arrivare a prendere una determinata zucca;

- numero minimo di zucche che ogni studente deve raccogliere.

Ricordando le attività svolte nelle prime lezioni (analisi delle caratteristiche che influenzano il funzionamento di una ruota idraulica e conseguente scelta di quelle ritenute essenziali), è stato richiesto agli studenti di proporre un elenco minimo di caratteristiche che descrivano la competizione, ai fini di calcolare la velocità media tenuta dalle squadre. L'insegnante ha sottolineato che serviva una rappresentazione semplificata della gara, sufficiente per raggiungere l'obiettivo di capire quanti metri si debbano percorrere per raccogliere un certo numero di zucche.

Sempre mediante una discussione collettiva si sono analizzate quindi le caratteristiche proposte per determinare quelle ridondanti e quelle necessarie. In questa fase l'insegnante ha cercato di orientare alcuni ragionamenti logici, anche riformulandoli nel caso non fossero stati esplicitati correttamente dagli studenti. 
Una caratteristica ridondante che è stata subito individuata, per esempio, è l'estensione del campo di gioco, in quanto si riesce a determinare dalle seguenti tre caratteristiche:

- distanza della prima zucca dallo start;

- distanza fra una zucca e l'altra;

- numero di zucche disposte su ogni fila.

Fra le caratteristiche non necessarie si è arrivati, tramite il vaglio delle proposte e delle relative spiegazioni in modalità collettiva, a includere anche le seguenti:

- numero di zucche disseminate per ogni fila (supponendo che tale numero sia comunque maggiore del numero di zucche che una squadra può raccogliere nel tempo prefissato);

- numero di concorrenti per squadra.

Dopo aver effettuato questa selezione si sono chiarite le caratteristiche considerate necessarie, ad esempio i metri da percorrere per arrivare a prendere una determinata zucca.

Per semplicità, è stato proposto inizialmente di trascurare la parte di percorso per riportare l'ultima zucca presa allo start, posticipando a un secondo momento la costruzione del modello completo, che considera cioè l'intero percorso per recuperare e portare allo start tutte le zucche prese.

Le caratteristiche necessarie per modellizzare matematicamente la situazione reale sono dunque risultate le seguenti:

- posizione della zucca rispetto le altre (prima, seconda, ...);

- distanza della prima zucca dallo start;

- distanza fra una zucca e l'altra;

- tempo disponibile per la gara;

- metri da percorrere per arrivare a prendere una determinata zucca.

2.3.2 Lezione 6: individuazione di variabili matematiche da associare alle caratteristiche dell'attività; prime semplificazioni e schematizzazioni

Gli studenti dovevano determinare le entità matematiche da associare alle caratteristiche individuate nella lezione precedente e distinguerle in parametri e variabili.

Gli studenti hanno riconosciuto subito che il tempo disponibile per la gara fosse da considerarsi un parametro, mentre hanno presentato qualche difficoltà per le altre caratteristiche e soprattutto nell'associarvi delle variabili matematiche. La docente ha poi esplicitato formalmente la relazione fra le caratteristiche dell'attività e le variabili matematiche, associando:

- la variabile $n$ alla zucca $n$-esima;

- il parametro d alla distanza della prima zucca dallo start;

- il parametro $l$ alla distanza fra una zucca e l'altra;

- il parametro $T$ al tempo disponibile per la gara;

- la variabile $a_{n}$ ai metri percorsi per prendere la zucca $n$-esima.

Proseguendo con la realizzazione dell'attività, i ragazzi sono stati sollecitati a individuare una relazione matematica fra le variabili.

Per far questo, gli studenti sono stati invitati a realizzare un modello grafico che schematizzasse l'attività, rimostrando loro i disegni rappresentativi delle ruote idrauliche (Figure 4 e 5). A questo scopo è stato consigliato di individuare un'alternativa all'oggetto "zucca" che fosse equivalente ai fini di descrivere in termini schematici l'attività della gara. Alla maggior parte degli studenti è venuta in mente la "palla". Si è dunque stabilito di considerare palle al posto di zucche.

La relazione matematica fra le variabili è di estrema importanza nella costruzione di un modello matematico, e individuare tale relazione è una fase fondamentale del processo, che mette in evidenza tutte le potenzialità offerte dalla modellizzazione matematica. 
Per far toccare con mano agli studenti l'importanza di individuare la relazione matematica fra le variabili, l'insegnante ha deciso di far prima calcolare empiricamente alcuni valori delle grandezze in gioco. Si è partiti da un esempio particolare, fissando i parametri $d$ e $l$ in modo che empiricamente gli studenti potessero ricavare i valori da attribuire alla variabile $a_{n}$ per $n=1,2,3,4,5$ e capire se questi valori potessero rappresentare i termini di una sequenza e, in caso affermativo, individuare la sequenza relativa (per poi definire la possibile relazione matematica fra $n$ e $a_{n}$ che si potrà poi estendere al caso di $d$ e $l$ generici).

Ponendo $d=3$ e $l=1$, tutte le palle sono poste alla distanza di un metro l'una dall'altra e la prima è posta alla distanza di 3 metri dallo start.

Gli studenti, divisi in piccoli gruppi, sono stati invitati a realizzare a casa dei possibili modelli grafici. Tornati in classe, i ragazzi hanno proposto i loro modelli grafici schematizzanti l'attività (tutti molto simili, qualcuno contemplava più file in parallelo; altri non descrivevano le grandezze associate). Dopo averli discussi collettivamente è stato adottato uno schema semplice e funzionale (Figura 8).

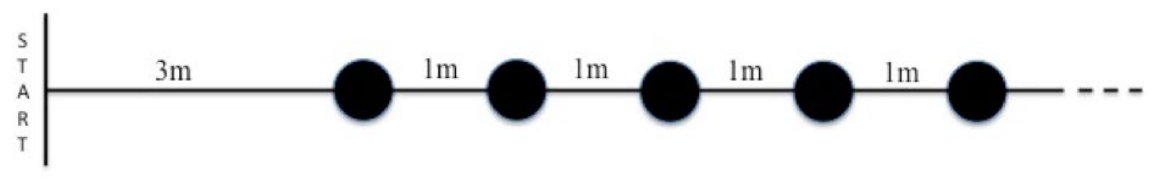

Figura 8. Schema della gara per l'esempio particolare.

\subsubsection{Lezione 7: ricerca di modi empirici per determinare i valori delle variabili matematiche a partire da casi particolari; analisi della relativa inefficienza}

Gli studenti sono stati invitati a calcolare empiricamente di volta in volta quanti metri i componenti della squadra vincitrice hanno dovuto percorrere per arrivare a prendere le prime palle (le prime cinque), ricordando che si prende una palla solo dopo che si è riportata indietro allo start la precedente. Sfruttando il disegno realizzato (Figura 8) gli studenti hanno facilmente trovato che: per arrivare a prendere la prima palla occorre percorrere tre metri; per prendere la seconda occorre prendere la prima, riportarla indietro allo start e poi avanzare fino alla seconda; per prendere la terza devono aver preso la seconda, riportata indietro e poi avanzato fino alla terza, e così via.

I ragazzi hanno inizialmente svolto i calcoli in modo informale, senza descriverne i passaggi. Sono stati poi invitati a riportare in dettaglio tutte le operazioni effettuate, per ottenere in termini matematici queste espressioni numeriche:

$a_{1}=3$

$$
\begin{aligned}
& a_{2}=3+3+4=10 \\
& a_{4}=19+5+6=30
\end{aligned}
$$

$$
\begin{aligned}
& a_{3}=10+4+5=19 \\
& a_{5}=30+6+7=43 .
\end{aligned}
$$

L'insegnante si è poi limitata a marcare i risultati delle espressioni proposte dagli studenti e a scrivere direttamente il numero di metri che occorre percorrere per prendere 6 palle, 7 palle aggiungendoli ai risultati precedenti:

$$
a_{6}=58 \quad a_{7}=75 .
$$

Quindi ha domandato agli studenti come potesse aver calcolato così rapidamente questi valori e se loro fossero in grado di calcolare i metri necessari per prendere l'ottava e la nona palla in modo rapido. Gli studenti inizialmente sono rimasti perplessi. Dopo diversi minuti uno studente è intervenuto dicendo il valore (corretto) $a_{8}=94$, mentre un altro studente ha subito fatto una controproposta: $a_{6}=57, a_{7}=73$ e ha ipotizzato $a_{8}=90$. 
La docente ha chiesto al secondo studente di esplicitare il suo ragionamento; ecco la risposta: «Si passa da un termine al successivo aumentando di uno le decine e ponendo come unità la somma delle cifre del termine precedente, se maggiore di 9 riconsidera la somma del numero ottenuto per le unità e aumenta ancora di uno le decine».

È stato proposto, a questo punto, di verificare quale dei due studenti avesse dato la risposta corretta rifacendo i calcoli come per i primi termini.

Gli studenti hanno quindi verificato che fosse corretta la proposta del primo. Alla richiesta di spiegazioni, alcuni studenti sono rimasti perplessi, altri invece hanno notato che la differenza di metri per prendere una zucca e quella precedente aumentava ogni volta di due:

$$
a_{2}-a_{1}=7 \quad a_{3}-a_{2}=9 \quad a_{4}-a_{3}=11 \quad a_{5}-a_{4}=13
$$

e correttamente hanno determinato: $a_{8}=94$ e $a_{9}=115$.

A questo punto si è arrivati collettivamente a capire che la sequenza numerica trovata non era casuale, ma guidata da una legge precisa espressa da molti studenti con queste parole: la differenza fra due risultati successivi di volta in volta è incrementata di due.

Gli studenti sono stati invitati a descrivere in termini matematici la legge che determina la sequenza individuata. L'insegnante ha fatto solo notare che si trattava di una successione definita in modo ricorsivo: il suo $n$-esimo termine è calcolato come funzione di alcuni dei precedenti, avendo posto delle condizioni iniziali per i primi termini. Ha poi chiesto di formalizzare la regola della sequenza individuata mediante una formula ricorsiva che esprimesse il termine $a_{n}$ come funzione dei due termini precedenti:

$$
a_{n}=f\left(a_{n-1}, a_{n-2}\right)
$$

poste le condizioni iniziali per i primi due termini $a_{1}=3$ e $a_{2}=10$.

Gli studenti, divisi in piccoli gruppi di lavoro, hanno cercato di determinare la funzione $f$, partendo dal descrivere in termini matematici quanto detto a parole, iniziando dalla differenza di metri percorsi rispettivamente per prendere la terza e la seconda palla. La maggior parte dei gruppi ha iniziato a scrivere: $a_{3}-a_{2}=\left(a_{2}-a_{1}\right)+2$. È stato loro ricordato che avrebbero dovuto calcolare i metri percorsi per prendere una determinata palla, nel modo più generico possibile e che le espressioni scritte sono espressioni algebriche per cui valgono le consuete regole algebriche.

I vari gruppi dopo esser arrivati a una scrittura di passaggio di questo tipo:

$$
a_{3}=a_{2}+\left(a_{2}-a_{1}\right)+2
$$

hanno determinato l'espressione seguente:

$$
a_{n}=a_{n-1}+\left[\left(a_{n-1}-a_{n-2}\right)+2\right]=2 a_{n-1}-a_{n-2}+2 .
$$

Gli studenti, in seguito, sono riusciti spontaneamente a calcolare in modo rapido altri termini della successione. A questo punto si è passati ad analizzare l'efficienza dei metodi utilizzati. L'insegnante ha invitato gli studenti a riflettere su come sia comunque lungo e laborioso calcolare i metri percorsi per prendere per esempio 40 palle, dato che i metri percorsi al passo $n$-esimo dipendono dai metri percorsi al passo $(n-1)$-esimo e $(n-2)$-esimo. Tramite una breve discussione collettiva, gli stessi studenti hanno concluso che i metri percorsi per cogliere sequenzialmente le varie palle formano una successione numerica e utilizzare una formula ricorsiva che la rappresenti non costituisce un metodo matematico efficiente per calcolare i metri percorsi nel caso di un numero di palle raccolte anche solo maggiore di qualche decina, caso che corrisponde alla situazione reale che si vuole descrivere. 


\subsubsection{Lezione 8: ricerca di una relazione diretta fra le variabili matematiche}

In questa lezione si è proposto agli studenti, divisi in gruppi, di trovare la relazione diretta fra $a_{n}$ ed $n$, necessaria per svincolarsi dall'idea di successione ricorsiva, in modo da poter poi generalizzare la costruzione del modello.

Alcuni gruppi sono partiti dalla formula ricorsiva e hanno provato ad applicarla per $n=3$. Hanno cercato di ricavare da quest'applicazione una regola generale ma non sono riusciti comunque a svincolarsi da una formula ricorsiva e quando sono stati invitati a verificarla nel caso di $n=4$ (qualcuno I'ha verificata autonomamente) hanno capito che non era la relazione cercata. In pratica, da $a_{1}=3, a_{2}=10$ e $a_{n}=2 a_{n-1}-a_{n-2}+2$, hanno messo in evidenza che $a_{3}=2 \cdot 10-3+2$ da cui hanno ipotizzato la relazione $a_{n}=(n-1) \cdot a_{n-1}-n+2$ che è sempre una formula ricorsiva.

Altri gruppi di studenti sono partiti dal cercare di generalizzare i calcoli empirici effettuati inizialmente per individuare i metri da percorrere per cogliere le prime palle:

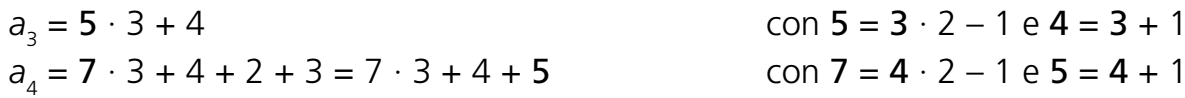

da cui hanno ipotizzato che $a_{n}=(2 n-1) \cdot 3+(n+1)$ salvo poi accorgersi che andava bene per $a_{3}$ ma che per $a_{4}$ mancava il termine $(+n)$.

\subsubsection{Lezione 9: definizione della relazione finale confacente alla situazione reale che si vuole de- scrivere con il modello}

Dopo aver lasciato del tempo a casa per effettuare qualche tentativo, solo un gruppo (di competenze eterogenee ma composto da due studenti con competenze avanzate) ha pensato di considerare la relazione cercata come somma di due espressioni, ciascuna corrispondente a una parte del moto.

La prima espressione corrisponde ai metri percorsi solo per ripetere la distanza dallo start alla prima palla: si tratta di 3 metri nel nostro caso, ripetuti per $2 n$ volte, meno i 3 metri per riportare allo start la palla $n$-esima; si ricorda che la docente ha suggerito di conteggiare inizialmente solo i metri percorsi per arrivare a prenderla. Si individua così l'espressione: $3(2 n-1)$.

La seconda espressione corrisponde ai metri percorsi ripetutamente dalla prima palla ad ogni palla successiva, fino alla palla $n$-esima: 1 metro dalla prima palla alla seconda palla (avanti e indietro), 2 metri dalla prima palla alla terza palla (avanti e indietro) e così via. Si ricava quindi l'espressione:

$$
1+1+2+2+\ldots+(n-3)+(n-3)+(n-2)+(n-2)+(n-1)
$$

che, addizionando il primo termine con il penultimo, il secondo con il terzultimo ecc., risulta un'addizione ripetuta di $(n-1)$ metri per $(n-1)$ volte:

$$
[1+(n-2)]+[1+(n-2)]+\ldots+(n-1)=(n-1)^{2} .
$$

Gli studenti hanno così presentato in classe la relazione corretta dopo averla verificata per svariati casi:

$$
a_{n}=3(2 n-1)+(n-1)^{2} \quad \text { ossia } \quad a_{n}=n^{2}+4 n-2
$$

La relazione è molto semplice ed esprimibile tramite un polinomio di secondo grado.

Prima di richiamare nuovamente l'attenzione sul gioco competitivo che si vuole rappresentare, l'insegnante ha chiesto a ogni studente della classe di controllare la validità della relazione finale, sia verificandola per svariati valori di $n$, sia dal punto di vista del procedimento utilizzato per definirla.

Il modello matematico individuato è utile per calcolare la velocità media della squadra vincitrice a partire dal numero di zucche, schematizzate come palle nel modello, che questa squadra ha preso. Però 
per individuare la relazione si è semplificata la situazione reale, fissando la distanza della prima zucca dalla posizione di start a 3 metri. La docente ha quindi chiesto agli studenti di provare a estenderla al caso $d$ qualsiasi (caso semi-generico) costruendo lo schema grafico relativo.

L'estensione al caso di d qualsiasi (Figura 9), non ha comportato particolari difficoltà:

$$
a_{n}=d(2 n-1)+(n-1)^{2} \quad \text { ossia } \quad a_{n}=n^{2}-2(1-d) n+1-d .
$$

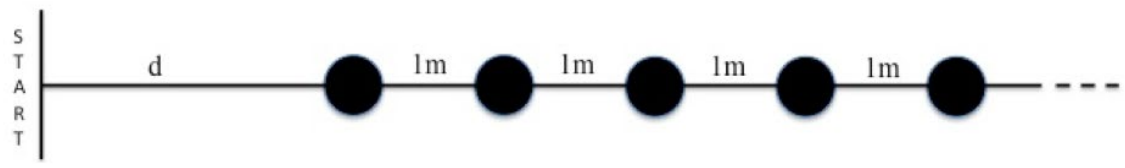

Figura 9. Schema gara semi-generico.

Un'altra semplificazione effettuata nell'approccio al modello è stata quella di calcolare i metri percorsi per arrivare a cogliere I'ultima palla, non includendo quindi quelli per riportarla allo start. Gli studenti, autonomamente, non hanno avuto difficoltà a trovare una relazione finale fra i metri effettivamente percorsi da una squadra vincitrice che abbia portato $n$ palle allo start, il numero di palle prese e la distanza parametrica $d$ della prima palla dallo start. Hanno dovuto semplicemente aggiungere alla relazione i metri da percorre per riportare la palla $n$-esima allo start, considerando che ciascuna è posta a distanza di un metro dalla successiva e che le palle precedenti l'ennesima sono $(n-1)$, dunque $(n-1)$ metri per tornare indietro a cui sommare $d$, dunque: $n-1+d$. Si è pertanto arrivati a definire la relazione finale:

$$
a_{n}=n^{2}-2(1-d) n+1-d+n-1+d \quad \text { o equivalentemente } a_{n}=n^{2}-n(1-2 d) .
$$

Se si sta lavorando con una classe brillante o se si vogliono stimolare gli studenti con competenze più avanzate, si può studiare il caso in cui anche la distanza fra le palle sia un numero qualsiasi di metri $l$ (caso generico) fissato a priori uguale per tutte (Figura 10).

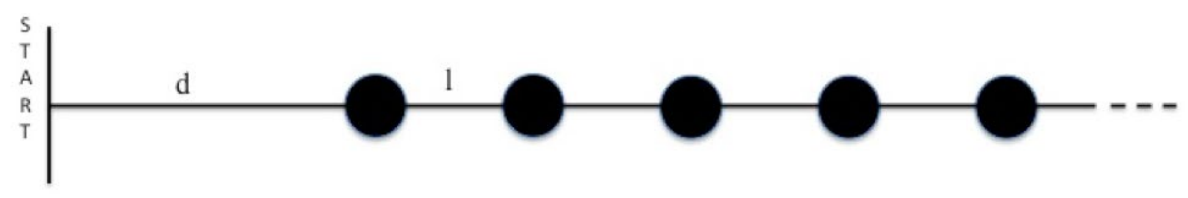

Figura 10. Schema gara caso generico.

In questa sperimentazione è stato accennato alla possibilità del caso generico ma non è stato affrontato.

\subsection{Fase conclusiva}

\subsubsection{Analisi del modello matematico generato}

Dopo aver determinato la relazione che esprime i metri percorsi in funzione del numero di palle prese, gli studenti sono stati invitati a soffermarsi ad analizzare il modello generato. Questa fase è stata guidata principalmente dall'insegnante, chiedendo la collaborazione degli studenti per arrivare a certe conclusioni una volta chiarite le premesse.

È stato ritenuto importante chiedere agli studenti di inquadrare la relazione nell'ambito del gioco competitivo che si è preso in esame all'inizio dell'esperienza didattica. Tale relazione è essenziale per calcolare la velocità media sostenuta da una squadra, conoscendo il parametro $T$, tempo di gara. Essenziale visto che il numero di zucche prese da ciascuna squadra vincitrice è sempre elevato e quindi altri metodi risulterebbero troppo onerosi nei calcoli. La stessa relazione può permettere, viceversa, di 
calcolare altrettanto rapidamente il numero di zucche/palle prese da chi ha percorso un certo numero di metri come risoluzione di una semplice equazione di secondo grado.

La possibilità di generalizzare la relazione rendendola valida per qualunque valore dei parametri $d$ e $l$ rende il modello generato flessibile, valido per tutte le possibili combinazioni di valori dei due parametri, rappresentanti altrettante situazioni reali. Riflettere su questa caratteristica può essere un'occasione per accennare alla struttura degli algoritmi dei programmi informatici alla base delle applicazioni che quotidianamente utilizziamo.

Nell'analizzare il modello costruito si è ritenuto importante far riflettere gli studenti sulla sua genesi, sui passaggi che hanno determinato proprio l'esigenza di individuarlo. Per gli studenti è stato anche un modo per ripercorrere le fasi del lavoro svolto e per interiorizzare meglio il significato stesso dell'esperienza. Per l'insegnante, invece, si è trattato della possibilità di valutare il successo o meno dell'attività didattica, quanto sia stata significativa dal punto di vista dell'apprendimento relativo e delle eventuali riflessioni che è riuscita a generare.

\subsubsection{Rielaborazione verbale strutturale dell'esperienza}

La rielaborazione è avvenuta in due fasi: la prima con modalità individuale orale, così da esercitare la comunicazione matematica e l'utilizzo di termini tecnici, anche suddividendo l'intera esperienza nelle sue fasi e facendole rielaborare da studenti diversi. La seconda modalità è stata scritta, schematica, attraverso un diagramma di flusso che è stato realizzato in piccoli gruppi (in modo che gli studenti condividessero quanto appreso e prestassero continuamente attenzione sia alle dinamiche interpersonali, sia al prodotto del lavoro).

Nelle rielaborazioni orali è emersa qualche difficoltà a utilizzare termini tecnici, ma in generale gli studenti sono riusciti a dare la giusta importanza alla necessità di semplificare la situazione reale per studiarla in termini matematici e all'efficacia di ricorrere a un modello matematico rappresentativo rispetto ad altri metodi di studio. Non molti invece hanno evidenziato personalmente l'analisi delle caratteristiche della situazione, ma spesso si sono limitati a citare la necessità di considerare le caratteristiche essenziali per poi trasformarle in variabili matematiche.

Si riporta di seguito il prodotto scritto finale di un gruppo (Figura 11). Nel corso della realizzazione gli studenti hanno chiesto varie conferme all'insegnante e, in caso di risposta negativa, si è suggerito di rivedere bene i loro appunti. L'imprecisione più frequente è stata di saltare almeno un passaggio, soprattutto I'analisi delle caratteristiche della situazione reale.

Inoltre, come si vede dal diagramma riportato (Figura 11), probabilmente per i termini di fatto utilizzati durante tutta l'esperienza, nonostante scrivano che «le zucche diventano palle» poi citano solo le zucche.
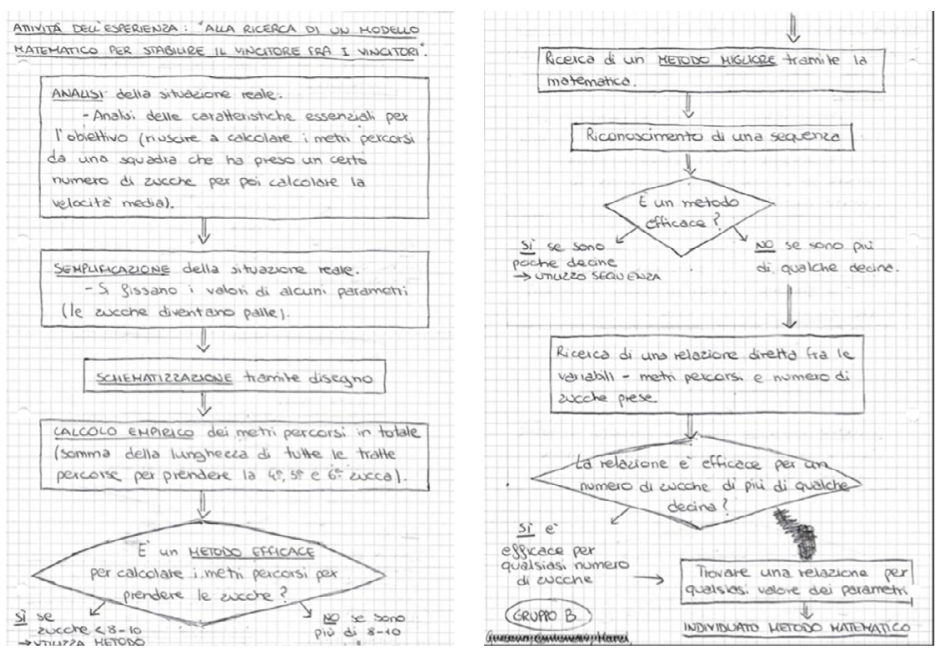

Figura 11. Schema riassuntivo dell'esperienza: prodotto finale di un gruppo di studenti. 


\subsection{Commento generale dell'insegnante sui risultati percepiti}

Le ultime due esperienze realizzate con queste premesse sono state svolte durante l'anno scolastico 2018-2019. In particolare, in una classe seconda (Allegato 2) e in una classe quarta del liceo scientifico, con risultati soddisfacenti.

La maggior parte degli studenti è riuscita a produrre in maniera autonoma quanto necessario per svolgere tutte le fasi dell'esperienza, dimostrando soprattutto una rielaborazione finale corretta.

In questa sperimentazione di costruzione di un modello matematico descrittivo di una situazione reale, l'insegnante ha dovuto guidare le relative fasi, ma gli studenti hanno prodotto in modo autonomo le schematizzazioni grafiche relative al gioco competitivo e soprattutto la relazione finale fra le variabili matematiche e i parametri associati alle caratteristiche fisiche della situazione reale. Una volta descritto il modello matematico (dai gruppi che l'hanno individuato a quelli che non sono riusciti a determinarlo) anche la relazione finale è stata comunque compresa da tutti.

In particolare, per la classe quarta, dall'analisi delle relazioni finali orali individuali e scritte di gruppo, e da alcune esercitazioni successive sulle sequenze, successioni ricorsive, funzioni parametriche e modelli matematici, effettuate dopo l'esperienza didattica descritta, i contenuti e le competenze disciplinari sembrano risultare per lo più acquisite dalla maggior parte degli studenti. Questa considerazione nasce dall'esito della correzione degli esercizi assegnati per casa o svolti in classe.

In particolare, per le sequenze numeriche e le funzioni parametriche l'insegnante ha riscontrato un livello di correttezza maggiore rispetto agli usuali risultati emersi nelle attività didattiche precedenti e soprattutto nella stessa classe prima dell'esperienza. Qualche esitazione sembra permanere a riguardo delle formule ricorsive.

\section{Conclusioni}

Superato ormai il primo ventennio del 2000, considerato l'enorme sviluppo e utilizzo dei modelli matematici in svariati campi del sapere umano, e considerato quanto contemplato dalle Indicazioni Nazionali, si potrebbe auspicare che la loro esplorazione entri definitivamente a far parte del bagaglio culturale degli studenti alla fine del loro percorso scolastico.

L'esempio di esperienza didattica descritto nell'articolo vuole rappresentare uno dei tanti possibili modi, efficaci e non eccessivamente complessi, di introdurre i modelli matematici e di far intuire agli studenti la loro efficienza e importanza. Le fasi introduttive dell'esperienza potrebbero costituire un ulteriore spunto di riflessione su quali requisiti di base gli studenti debbano possedere prima di affrontare il concetto di modellizzazione matematica. Inoltre, l'esperienza di costruire un modello matematico, per quanto semplice, se avviene prima di affrontare modelli precostituiti probabilmente creerà un inizio di familiarità che renderà meno meccanico e fine a sé stesso l'utilizzo successivo di modelli precostituiti.

La validità dell'attività didattica descritta può essere al momento dedotta dai risultati percepiti dall'insegnante nelle ultime sperimentazioni effettuate in licei scientifici (par. 2.5). Probabilmente l'esperienza potrebbe dare risultati migliori, se si proponesse prima qualche semplice esempio di costruzione di un modello. Ad esempio, si potrebbe utilizzare come premessa all'esperienza quanto riportato nell'Allegato 1, che evidenzia come un modello matematico ricerchi sempre un legame funzionale fra le variabili e non sia interessato a esplicitare risultati di calcoli particolari, validi solo per determinati valori dei dati. Per quanto riguarda l'introduzione teorica, potrebbe essere più efficace effettuarla in chiusura dell'attività, per formalizzare e generalizzare quanto appreso durante l'esperienza. II concetto di modello matematico, quindi, verrebbe formalizzato dopo che gli studenti hanno avuto la possibilità di sperimentarne un esempio nelle varie fasi dell'attività stessa. Si potrebbe chiedere loro di formulare una definizione formale da assegnare ai modelli matematici e poi confrontare le loro enunciazioni con le definizioni storiche. Probabilmente l'attenzione in classe sarebbe più viva e gli 
studenti potrebbero più facilmente interiorizzare la maggior parte delle informazioni fornite. Inoltre, nei tentativi di trovare la relazione finale rappresentativa del modello, gli studenti sono stati fuorviati dal cercare di utilizzare la formula ricorsiva dedotta in precedenza. In questo caso particolare sarebbe stato più semplice non tenerne conto. L'idea di evidenziare la formula ricorsiva, dopo aver individuato la sequenza, nasce da un'esigenza di completezza. Anche in questo caso però forse sarebbe meglio svolgere questo passaggio dopo aver trovato la relazione finale.

Questa esperienza, pensata per introdurre la modellizzazione matematica nella scuola secondaria di secondo grado, risulta guidata in varie parti dall'insegnante anche se, con il raffinamento della proposta da parte dell'insegnante nel corso degli anni, gli studenti sono riusciti a svolgere autonomamente dei passi importanti (come individuare la relazione finale fra le grandezze rappresentative della situazione, analizzare le suddette grandezze, schematizzare e semplificare la situazione). L'esperienza didattica potrebbe proseguire con la stessa classe, proponendo altri esempi di costruzione di un modello matematico, diminuendo di volta in volta la guida dell'insegnante.

Rimane da sperimentare se sia più efficace cercare prima uno schema semplificativo nel caso generico e poi assegnare valori ai parametri, per trovare in un primo momento in modo più facile una relazione tra le variabili, e se sia meglio schematizzare la situazione reale prima di definire le caratteristiche essenziali in modo che la semplificazione della situazione sia facilitata dai tentativi di realizzazione dello schema grafico. La seconda proposta potrebbe essere efficace per definire meglio le caratteristiche essenziali. Un altro punto aperto è come formare i gruppi nei vari lavori: gruppi omogenei per livello di competenze in modo da permettere a tutti i componenti del gruppo di rispettare più o meno i propri tempi di riflessione, o gruppi eterogenei in modo da stimolare la collaborazione tra studenti con competenze di livello differente, inducendoli a trarre giovamento dalla situazione sia per esplicitare le proprie difficoltà sia per chiarire le proprie strategie.

A posteriori, sarebbe stato interessante confrontare gli schemi riassuntivi dell'esperienza, prodotti finali dei vari gruppi di studenti, eventualmente in modalità collettiva; spesso tali schemi risultano incompleti, ma dalla loro unione e confronto si potrebbe ottenere una relazione schematica esaustiva dell'attività, ricca anche di spunti di riflessione.

Sarebbe interessante esaminare ulteriori risultati, in altri tipi di scuole e di realtà, e confrontare eventualmente altri metodi aggiuntivi e/o sostitutivi. Al momento si ha a disposizione solo quanto svolto successivamente in un'altra classe quarta, sempre liceo scientifico, di una scuola sita in una grande periferia. In particolare, uno studente con competenze avanzate, dopo l'individuazione della prima sequenza numerica, ha ricavato (in un pomeriggio a casa) direttamente la relazione generale fra i metri percorsi (indicati questa volta con $z_{n^{\prime}}$ scelta della classe) e il numero di zucche prese, considerando qualsiasi distanza $l$ fra una zucca e la successiva e qualsiasi distanza $d$ fra la prima zucca e lo start. II procedimento utilizzato, riportato in Figura 12, è interessante e originale.

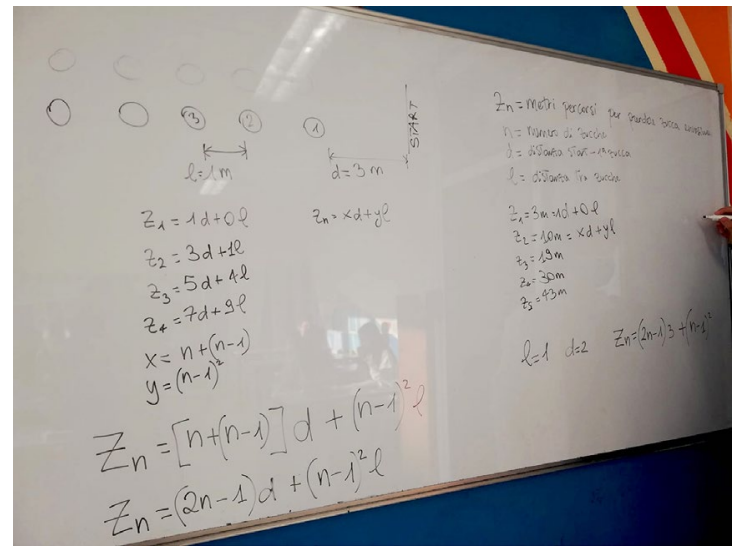

Figura 12. Relazione finale caso generico. 
La determinazione di sequenze, di formule ricorsive durante lo svolgimento della costruzione del modello rappresenta un'occasione per evidenziare come la matematica offra spesso diversi modi per arrivare a determinare lo stesso risultato, tutti validi, logicamente corretti, seppur diversi dal punto di vista dell'efficienza. L'ottimizzazione che scaturisce proprio dalla scelta risolutiva basata sul loro confronto potrebbe essere uno spunto di riflessione e ampliamento dei concetti trattati.

L'esempio, modulare nella sua difficoltà, che contempla un particolare utilizzo di grandezze parametriche, apre una visione, oltre che sui modelli matematici, anche sulla struttura degli algoritmi dei programmi informatici alla base di applicazioni che quotidianamente utilizziamo.

L'esperienza didattica presentata nell'articolo potrebbe dunque essere utile anche per ampliare l'orizzonte matematico degli studenti.

\section{Bibliografia}

Accademia dei Lincei. (2019). La genesi dei modelli: teoria, simulazioni e dati. https://sites.google.com/view/ modellilincei2019/

Bergamini, M., Barozzi, G., \& Trifone, A. (2015). Verso la seconda prova di matematica. Zanichelli.

Bergamini, M., Barozzi, G., \& Trifone, A. (2017). 5 Matematica.Blu 2.0. Zanichelli.

Comincioli, V. (2004). Problemi e Modelli matematici nelle Scienze Applicate. Ambrosiana.

Israel, G. (2003). La visione matematica della realtà. Laterza.

Ministero dell'Istruzione, dell'Università e della Ricerca. (2010). Schema di regolamento recante "Indicazioni nazionali riguardanti gli obiettivi specifici di apprendimento concernenti le attività e gli insegnamenti compresi nei piani degli studi previsti per i percorsi liceali". http://www.indire.it/lucabas/lkmw file/licei2010/indicazioni nuovo impaginato/ decreto indicazioni nazionali.pdf

Natalini, R. (2013). I modelli matematici: dai laboratori di ricerca alle aule scolastiche. http://maddmaths.simai. eu/divulgazione/scuola/materiali-relativi-al-ix-convegno-corso-di-formazione-i-modelli-matematici-dai-laboratori-di-ricerca-alle-aule-scolastiche/

Sasso, L., \& Zanone, C. (2019). Colori della matematica. Edizione Blu, Volume 5 a $\beta$. Petrini. 